\title{
Steroid use in elderly critically ill COVID-19 patients
}

\author{
To the Editor: \\ Copyright @The authors 2021 \\ This version is distributed under \\ the terms of the Creative \\ Commons Attribution \\ Non-Commercial Licence 4.0. \\ For commercial reproduction \\ rights and permissions contact \\ permissions@ersnet.org \\ Received: 6 April 2021 \\ Accepted: 2 June 2021

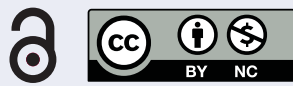 \\ More than a year after the onset of the severe acute respiratory syndrome coronavirus 2 (SARS-CoV-2) \\ pandemic, treating patients with coronavirus disease 2019 (COVID-19) remains a challenge. In contrast to \\ the rapid development of effective vaccines against SARS-CoV-2, the development of specific and \\ effective therapeutics against COVID-19 remains largely unresolved. \\ In addition to standard intensive care, including oxygen therapy and organ support when required, the use \\ of systemic corticosteroids was found to have a positive effect in randomised trials. However, data \\ regarding treatment of elderly COVID-19 patients are scarce. \\ Importantly, treatment with corticosteroids has well documented deleterious effects [1]: While the \\ immunosuppressive effect in patients with COVID-19 is presumably responsible for the desired therapeutic \\ effect, it may also render the patients more prone to secondary bacterial infections and potentially decrease \\ viral clearance [2]. Corticosteroid therapy is also associated with hyperglycaemia, has catabolic effects and \\ is associated with neuropathy. This could potentially affect the risk-benefit balance, especially in \\ vulnerable patient groups, such as elderly, frail patients. \\ The aim of this secondary analysis was to investigate the effects of corticosteroid therapy in an \\ international observational prospective study of critically ill elderly patients with COVID-19. \\ The COVIP study (“Corona Virus disease (COVID19) in Very Elderly Intensive care Patients (VIPs)”; \\ NCT04321265) included patients aged 70 years or older with proven COVID-19 and admitted to an \\ intensive care unit (ICU) [3]. 30-day mortality was defined as the primary endpoint. The study was \\ conducted by the Very old Intensive care Patient (VIP) network [4] across 207 ICUs in 35 countries. Data \\ were collected through an electronic case report form. A prospective study design was chosen to achieve \\ high-quality data. Informed consent was taken if not waived by the local ethical committee. \\ Two multi-level logistic regression models were utilised: the first model used the hospital unit as random \\ effect and the steroid use as fixed effect; the second model was a multi-variable model adjusting for "The \\ Sequential Organ Failure Assessment” (SOFA) score and frailty as assessed by the Clinical Frailty Scale. \\ Sensitivity analyses complemented the analysis. \\ In total, 3082 patients were included in the COVIP study; 2115 patients received corticosteroids, and 967 \\ patients received none. Median age was 75 (interquartile range (IQR) 72-79) years in both groups. With a \\ median SOFA score of 5 (IQR 3-8), there was no difference between the two groups. \\ 30-day mortality was $53 \%$ in the group treated with corticosteroids and $42 \%$ in the no-corticosteroid group \\ $(\mathrm{p}<0.001)$. \\ The univariate 30-day mortality rates were higher in patients receiving corticosteroids (53 versus 42\%; \\ aOR 1.16, 95\%CI 1.28-2.02; $\mathrm{p}<0.001$ ). This association of corticosteroid use was even more pronounced \\ after 3 months (69\% versus 49\%; $\mathrm{p}<0.001$; figure $1 \mathrm{a}$ ). In addition, we found that corticosteroids remained \\ associated with increased odds of 30-day mortality after multivariable adjustment (aOR 1.60, 95\% CI \\ $1.26-2.04$; $<0.001$ ). Further sensitivity analyses consistently confirmed the finding in subgroups stratifying \\ Shareable abstract (@ERSpublications) \\ This secondary analysis of the COVIP study shows a higher 30-day mortality in critically ill elderly \\ COVID-19 patients who received steroids as part of their treatment @cjungMD https://bit.ly/3xdyEur \\ Cite this article as: Jung C, Wernly B, Fjølner J, et al. Steroid use in elderly critically ill COVID-19 \\ patients. Eur Respir J 2021; 58: 2100979 [DOI: 10.1183/13993003.00979-2021].
}




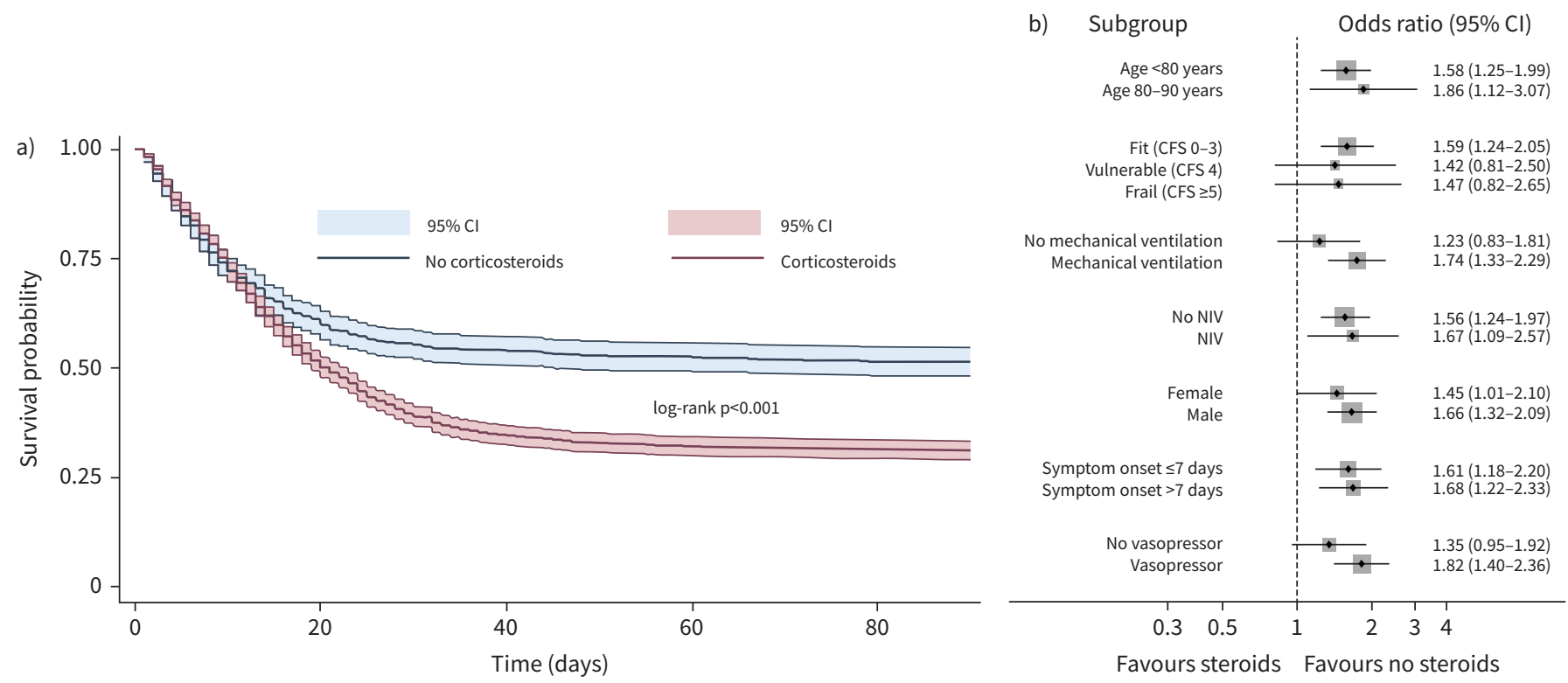

FIGURE 1 a) Plot of the Kaplan-Meier estimator illustrating survival probability up to 90 days after admission to the intensive care unit for patients with and without corticosteroid treatment. Log-rank-test: $p<0.001$. b) Sensitivity analyses stratifying 30-day mortality in subgroups for patient-specific characteristics and management strategies using multi-level logistic regression models. We depicted adjusted odds ratios from the model with the hospital unit as random effect and steroid use as fixed effect. CFS: Clinical Frailty Scale; NIV: noninvasive ventilation.

for age $(<80 / \geqslant 80$ years), frailty (fit/vulnerable/frail), mechanical ventilation (yes/no), noninvasive ventilation (yes/no), sex (female/male), symptom onset ( $\leqslant 7$ days/ $>7$ days), and vasopressor therapy (yes/no) (figure 1b). Furthermore, in sensitivity analyses evaluating patients in the first surge (March-May, aOR 1.38, 95\% CI 1.05-1.82; $\mathrm{p}=0.02 ; \mathrm{n}=1448$ ) and the second surge (September-December, aOR 2.09, 95\% CI $1.04-4.21 ; \mathrm{p}=0.04 ; \mathrm{n}=1414$ ) the finding was sustained.

In this prospective study of more than 3000 critically ill COVID-19 patients aged 70 years and older, we have found an independent association of steroid use with increased mortality.

These results question the routine use of corticosteroid treatment in elderly COVID-19 patients. While the immunosuppressive effect of steroids is undisputed and desirable in the context of severe COVID-19 treatment, the adverse effects of steroid treatment in elderly patients may outweigh the potential benefits.

This is the largest prospective analysis of critically ill elderly patients in relation to corticosteroid use to treat severe COVID-19 disease. Corticosteroid therapy has been established as standard of care in all ICU patients. However, even landmark randomised controlled trials do not support this with evidence in elderly patients. The RECOVERY study [5] showed no effect of corticosteroids in their subgroup of patients $>70$ years. Of note, only 169 patients in this group were on mechanical ventilation. The CoDEX study found no effect on mortality after 28 days, but no data was supplied specifically looking at patients above 70 years old [6]. Thus, both studies included far fewer patients than our current analysis. It is important to note that our data does not question the corticosteroid strategy in younger COVID-19 patients. It just emphasises that the decision to use corticosteroids needs to be individually tailored, first and foremost according to age, but also with regards to comorbidities and other factors [7].

Our analysis has limitations: First, this is a secondary analysis of a prospective study. Second, our study is not randomised and, despite multivariable adjustment, it is likely that unknown confounding factors may have contributed to our findings. Third, we have no detailed information about dosage and duration of corticosteroid treatment.

In conclusion, in this prospective observational study we found a higher 30-day mortality in critically ill elderly COVID-19 patients who received steroids as part of their treatment. 
Christian Jung $\oplus^{1}$, Bernhard Wernly ${ }^{2}$, Jesper Fjølner ${ }^{3}$, Raphael Romano Bruno ${ }^{1}$, David Dudzinski ${ }^{4}$, Antonio Artigas ${ }^{5}$, Bernardo Bollen Pinto ${ }^{6}$, Joerg C. Schefold ${ }^{7}$, Georg Wolff ${ }^{1}$, Malte Kelm $\oplus^{1}$, Michael Beil ${ }^{8}$, Sviri Sigal ${ }^{8}$, Peter Vernon van Heerden ${ }^{9}$, Wojciech Szczeklik ${ }^{10}$, Miroslaw Czuczwar $\circledast^{11}$, Muhammed Elhadi ${ }^{12}$, Michael Joannidis ${ }^{13}$, Sandra Oeyen ${ }^{14}$, Tilemachos Zafeiridis ${ }^{15}$, Brian Marsh ${ }^{16}$, Finn H. Andersen ${ }^{17,18}$, Rui Moreno $\oplus^{19}$, Maurizio Cecconi $\circledast^{20}$, Susannah Leaver ${ }^{21}$, Ariane Boumendil ${ }^{22,23}$, Dylan W. De Lange ${ }^{24}$, Bertrand Guidet ${ }^{22,23}$, Hans Flaatten ${ }^{25,26}$ and the COVIP study group

${ }^{1}$ Medical Faculty, Dept of Cardiology, Pulmonology and Vascular Medicine, Heinrich-Heine-University Duesseldorf, Duesseldorf, Germany. ${ }^{2}$ Dept of Anaesthesiology, Perioperative Medicine and Intensive Care Medicine, Paracelsus Medical University, Salzburg, Austria. ${ }^{3}$ Dept of Intensive Care, Aarhus University Hospital, Aarhus, Denmark. ${ }^{4}$ Cardiac Intensive Care Unit, Massachusetts General Hospital, Boston, MA, USA. ${ }^{5}$ Dept of Intensive Care Medicine, CIBER Enfermedades Respiratorias, Corporacion Sanitaria Universitaria Parc Tauli, Autonomous University of Barcelona, Sabadell, Spain. ${ }^{6}$ Dept of Acute Medicine, Geneva University Hospitals, Geneva, Switzerland. ${ }^{7}$ Dept of Intensive Care Medicine, Inselspital, Universitätsspital, University of Bern, Bern, Switzerland. ${ }^{8}$ Dept of Medical Intensive Care, Hadassah Medical Center and Faculty of Medicine, Hebrew University of Jerusalem, Jerusalem, Israel. ${ }^{9}$ Dept of Anesthesia, Intensive Care and Pain Medicine, Hadassah Medical Center and Faculty of Medicine, Hebrew University of Jerusalem, Jerusalem, Israel. ${ }^{10}$ Center for Intensive Care and Perioperative Medicine, Jagiellonian University Medical College, Krakow, Poland. ${ }^{11}$ 2nd Dept of Anesthesiology and Intensive Care, Medical University of Lublin, Lublin, Poland. ${ }^{12}$ Faculty of Medicine, University of Tripoli, Tripoli, Libya. ${ }^{13}$ Division of Intensive Care and Emergency Medicine, Dept of Internal Medicine, Medical University Innsbruck, Innsbruck, Austria. ${ }^{14}$ Dept of Intensive Care $1 \mathrm{~K} 12 \mathrm{IC}$, Ghent University Hospital, Ghent, Belgium. ${ }^{15}$ Intensive Care Unit General Hospital of Larissa, Larissa, Greece. ${ }^{16}$ Mater Misericordiae University Hospital, Dublin, Ireland. ${ }^{17}$ Dept Of Anaesthesia and Intensive Care, Ålesund Hospital, Ålesund, Norway. ${ }^{18}$ Dept of Circulation and Medical Imaging, Norwegian University of Science and Technology, Trondheim, Norway. ${ }^{19}$ Unidade de Cuidados Intensivos Neurocríticos e Trauma, Hospital de São José, Centro Hospitalar Universitário de Lisboa Central, Faculdade de Ciências Médicas de Lisboa, Nova Médical School, Lisbon, Portugal. ${ }^{20}$ Dept of Anaesthesia IRCCS, Instituto Clínico Humanitas, Humanitas University, Milan, Italy. ${ }^{21}$ General Intensive Care, St George's University Hospitals NHS Foundation Trust, London, UK. ${ }^{22}$ Sorbonne Universités, UPMC Univ Paris 06, INSERM, UMR_S 1136, Institut Pierre Louis d'Epidémiologie et de Santé Publique, Equipe: épidémiologie hospitalière qualité et organisation des soins, Paris, France. ${ }^{23}$ Assistance Publique - Hôpitaux de Paris, Hôpital Saint-Antoine, Service de réanimation médicale, Paris, France. ${ }^{24}$ Dept of Intensive Care Medicine, University Medical Center, University Utrecht, Utrecht, The Netherlands. ${ }^{25}$ Dept of Clinical Medicine, University of Bergen, Bergen, Norway. ${ }^{26}$ Dept of Anaesthesia and Intensive Care, Haukeland University Hospital, Bergen, Norway.

Corresponding author: Christian Jung (christian.jung@med.uni-duesseldorf.de)

This study is registered at Clinicaltrials.gov as NCT04321265.

Conflict of interest: C. Jung has nothing to disclose. B. Wernly has nothing to disclose. J. Fjølner has nothing to disclose. R.R. Bruno has nothing to disclose. D. Dudzinski has nothing to disclose. A. Artigas reports grants from Grifols and Fisher\&Paykel, personal fees for advisory board work from Grifols, Novartis and Lilly Foundation, outside the submitted work. B. Bollen Pinto has nothing to disclose. J.C. Schefold has nothing to disclose. G. Wolff has nothing to disclose. M. Kelm has nothing to disclose. M. Beil has nothing to disclose. S. Sigal has nothing to disclose. P.V. van Heerden has nothing to disclose. W. Szczeklik has nothing to disclose. M. Czuczwar has nothing to disclose. M. Elhadi has nothing to disclose. M. Joannidis has nothing to disclose. S. Oeyen has nothing to disclose. T. Zafeiridis has nothing to disclose. B. Marsh has nothing to disclose. F.H. Andersen has nothing to disclose. R. Moreno has nothing to disclose. M. Cecconi has nothing to disclose. S. Leaver has nothing to disclose. A. Boumendil has nothing to disclose. D.W. De Lange has nothing to disclose. B. Guidet has nothing to disclose. H. Flaatten has nothing to disclose.

Support statement: This work was supported by the European Commission, APHP and Health Region West (Norway). Funding information for this article has been deposited with the Crossref Funder Registry.

\section{References}

1 Waterer GW, Rello J. Steroids and COVID-19: we need a precision approach, not one size fits all. Infect Dis Ther 2020; 9: 701-705. 
2 Britt RC, Devine A, Swallen KC, et al. Corticosteroid use in the intensive care unit: at what cost? Arch Surg 2006; 141: 145-149.

3 Jung $\mathrm{C}$, Flaatten $\mathrm{H}$, Fjolner J, et al. The impact of frailty on survival in elderly intensive care patients with COVID-19: the COVIP study. Crit Care 2021; 25: 149.

4 Guidet B, de Lange DW, Boumendil A, et al. The contribution of frailty, cognition, activity of daily life and comorbidities on outcome in acutely admitted patients over 80 years in European ICUs: the VIP2 study. Intensive Care Med 2020; 46: 57-69.

5 Horby P, Lim WS, Emberson JR, et al. Dexamethasone in hospitalized patients with Covid-19. N Engl J Med 2021; 384: 693-704.

6 Tomazini BM, Maia IS, Cavalcanti AB, et al. Effect of dexamethasone on days alive and ventilator-free in patients with moderate or severe acute respiratory distress syndrome and COVID-19: the CoDEX randomized clinical trial. JAMA 2020; 324: 1307-1316.

7 Gogali A, Kyriakopoulos C, Kostikas K. Corticosteroids in COVID-19: one size does not fit all. Eur Respir J 2021; 57: 2100224. 